\title{
RETRACTED
}

\section{Targeting gene-virotherapy of cancer}

\author{
Xin Yuan $\mathrm{Liu}^{1,2}$, Jin $\mathrm{Fa} \mathrm{Gu}{ }^{1}$ \\ ${ }^{1}$ Institute of Biochemistry and Cell Biology, Shanghai Institutes for Biological Sciences, Chinese Academy of Sciences, 320 Yue \\ Yang Road, Shanghai 200031, China; ${ }^{2}$ Xinyuan Institute of Medicine and Biotechnology, School of Life Science, Zhejiang Sci-Tech \\ University, Hangzhou, 310018, China
}

Our purpose is to completely elimination of xenograft tumor in animal tumor model in order to work out a protocal for the cure of patient. Gene therapy and viral therapy for cancer have got some therapeutic effects, but both have no great breakthrough. Therefore, we worked out a new strategy called Targeting Gene-Virotherapy of Cancer which is a combination of the advantage of gene therapy and virotherapy. This new strategy has stronger antitumor effect than either of them alone. A tumor specific replicative adenovirus vector ZD55 (E1B 55KD deleted Adv.) which is similar to ONYX-015 in targeting fuction but significant different in construction was produced and various single therapeutic gene was inserted into ZD55. Now such a conception as Targeting Gene-Virotherapy of Cancer was raised and systemically studied before, although there are some works on ONYX-015-tk, -cd or cd/-tk etc. separately. The antitumor effect of ZD55-Gene (for example IL-24 gene) is much better than ZD55 (virotherapy) alone and hundred fold high than that of Ad-IL-24 (gene therapy) alone. ZD55-IL-24 was in preclinal studying in the ZD55-IL-24 therapy, completely elimination of tumor mass was occurred in some mice but not in all mice, that means one gene was not effictive enough to eliminate all the tumor mass in all mice. Therefore two genes with compensative or synergetic effect were inserted into ZD55 sepearately and used in combinction. This strategy was called Targeting Dual Gene-Virotherapy of Cancer (with PCT patent). Then much better results were obtained and all the xenograft tumor masses were completely eliminated in all mice, if two suitable genes were chosen. On the basis of the initiation of two gene results, it was thought about that using two tumors promoter to control the virus vector will be better for the targeting effect and the safty of the drugs. Then double tumor controlled virus vector harboring two genes for cancer therapy was worked out. Better results have been obtained and another patent has been applied. This antitumor strategy could be used to kill all the tumor cells completely in all mice with minimum damage to normal cells.

Cell Research (2006) 16:25-30. doi:10.1038/sj.cr.7310005; published online 16 January 2006

Keywords: targeting therapy, cancer therapy, gene-virotherapy

The use of gene therapy and virotherapy (the use of viruses as a form of treatment) [1] have contributed significantly to the treatment of cancer, though neither technology has proven to be very successful. Our group has combined the benefits of both technologies to develop a new method (Targeting Gene-Virotherapy) that improved the efficacy of tumor elimination. The targeting effect of virotherapeutics is retained while the weak toxic effects of viral vaccines

Correspondence: Xin Yuan Liu

Tel: 86-021-54921127; Fax: 86-021-54921126;

E-mail: xyliu@sibs.ac.cn were enhanced by the expression of antitumor transgenes. These results were published recently [2]. In 2000, only a few scientists used similar strategies. Though this strategy has not yet gained widespread use, its use should become greater in the future due to its benefits over the previous first-generation therapies (Table 1).

\section{ZD55-System and its targeting dual gene-virotherapy system}

ZD55 is a modified adenovirus similar to ONYX-015 (Adenovirus E1B 55KDa gene deleted) but with significant 
Table 1 Comparison of gene therapy and gene-virotherapy

\begin{tabular}{lll}
\hline Gene Therapy & Virotherapy & Gene-Virotherapy \\
\hline No cancer specific targeting & Having cancer specific targeting & Having cancer specific targeting \\
Lower infectivity and lower & High infectivity, no antitumor gene & Higher infectivity and highly \\
expression of gene & expressed & gene expressed 100 to 1000 fold \\
Lower killing effect to cancer & Lower killing effect to cancer & Higher killing effect to cancer \\
\hline
\end{tabular}
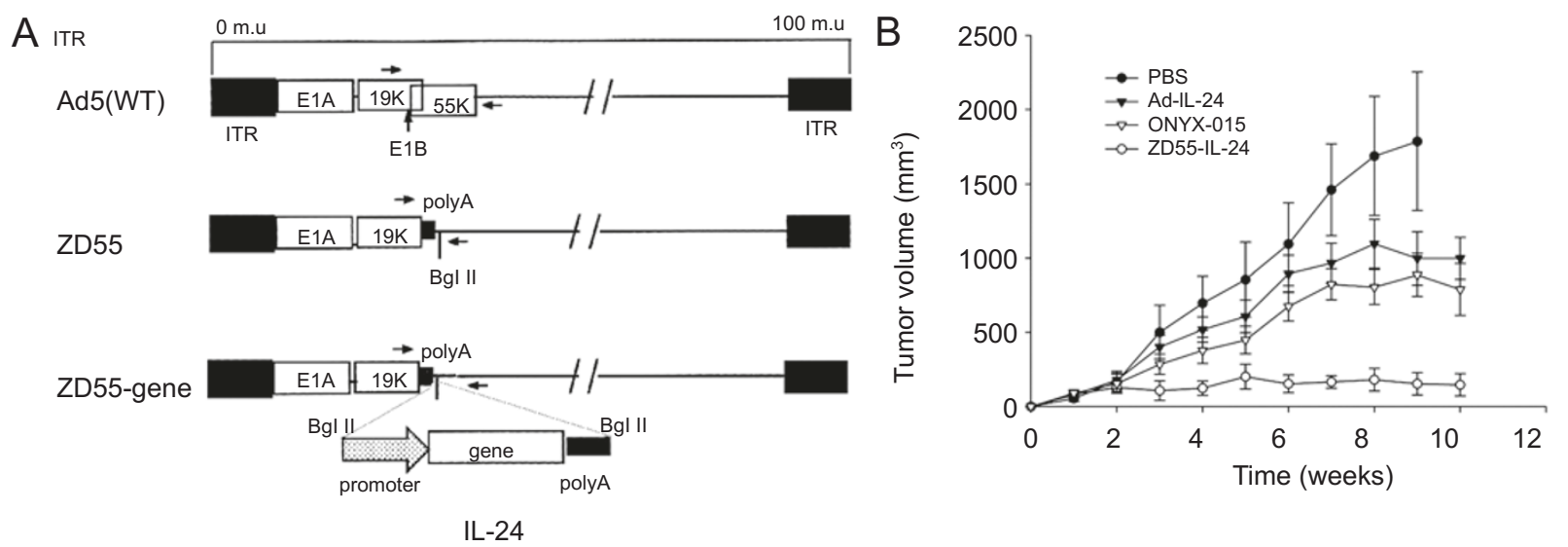

Figure 1 Construction of ZD55-gene for antitumor therapy. (A) Lane1, Wild type Adenovirus. Lane2, Delete 55KD gene, of adenovirus E1B and create a Bgl II site. Lane3, Insert an IL-24 expression cassette controlled by CMV promoter. (B) Antitumor effect of IL-24.

differences. ONYX-015 is a fusion virus of Adv2/Adv5, while ZD55 has elements of only Adv5. Furthermore, ONYX-015 has no convenient restriction endonuclease sites to insert foreign antitumor gene, while ZD55 has one cloning site to insert recombinant genes. Over ten genes have been inserted into ZD55 to form ZD55-gene constructs such as ZD55-Trail, ZD55-Smac, ZD55-XAF-1, ZD55-K5, ZD55-VEGI, ZD55-sFlt-1, ZD55-IL-2, ZD55IL-12, ZD55-IL-24, ZD55-MnSOD, and ZD55-CYLD. The antitumor effects of these recombinant viruses were better either than ZD55 alone, ONYX-015 (Virotherapy) alone or Ad-gene (Gene therapy) alone. In this minireview we focus on data from ZD55-IL-24 and ZD55-Trail recombinant viruses.

The ZD55-IL-24 was constructed as in Figure 1A and the antitumor effect was shown in Figure 1B [3].

We anticipate that ZD55-based virotherapeutics would be approved for clinical use because the similar virotherapeutic ONYX-15 had endured Phase III Trials by the FDA without overt cytotoxicity and the recombinant adenovirus H101 that is similar to ONYX-015 or ZD55 had been approved for marketing by SFDA (Chinese FDA). We anticipate excellent success with ZD55-IL-24 in clinical trials because the less-effective Ad-IL-24 virotherapeutic

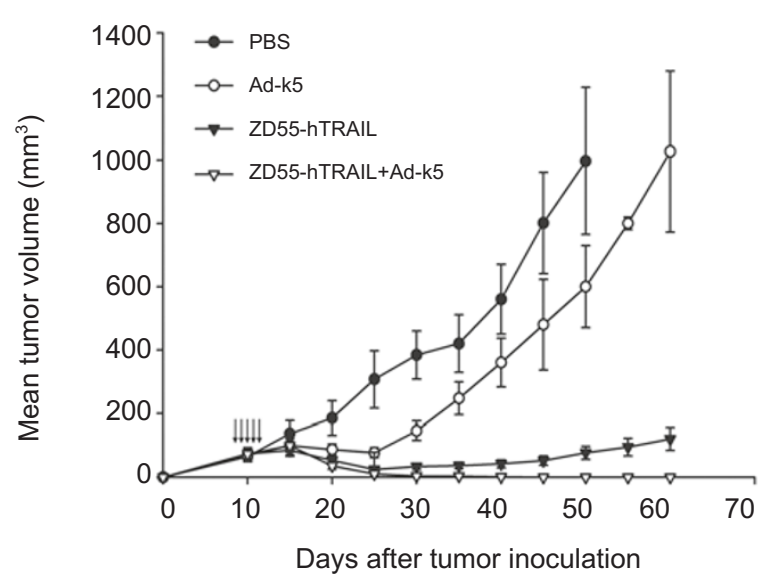

Figure 2 Antitumor effect of combined ZD55-Trail and Ad-K5.

has succeeded Phase II clinical trials in the USA, and preclinical studies of ZD55-IL-24 are so far promising.

\section{Targeting dual gene-virotherapy of cancer}

One ZD55-Gene, sometimes can completely eliminate all the tumor mass in some mice, but never in all mice. If two ZD55-genes are used, we completely and consistently 


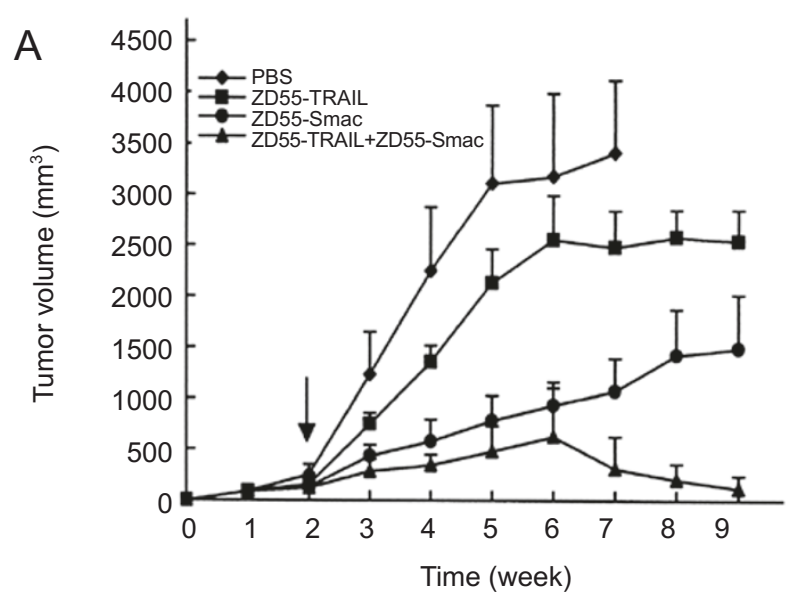

B

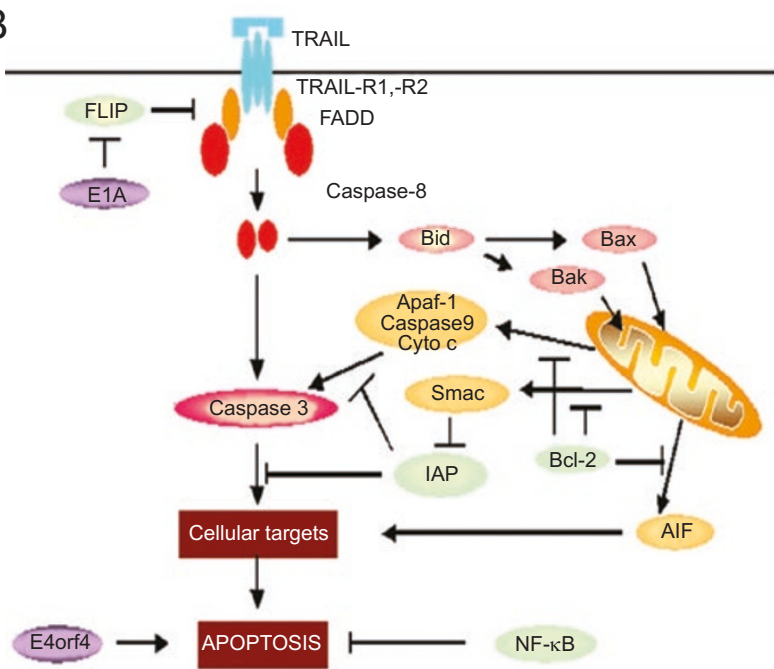

Figure 3 (A) Antitumor effect of combined ZD55-Trail and ZD55Smac. (B) A diagram to show the synergic effect of Trail and Smac.

eliminated of the tumor mass in all mice; this likely occurs because the two genes have synergistic of compensatory effects.

One pair of virotherapeutics that we tested was ZD55Trail and Ad (CA13)-K5. The K5 is the kringle 5 domain of plasminogen; its anti-angiogenic effect is better than K1-3 and that of angiostatin itself (K1-4). When tumors

Figure 4 Studies on the antitumor effect of MnSOD and its action mechanism. (A) Induce of MnSOD expression by ZD55-Trail in SW620 cell. (B) Antitumor effect of combined use of ZD55-MnSOD and ZD55-Trail. (C) New pathway for action of MnSOD in the induction of Bax by $\mathrm{H}_{2} \mathrm{O}_{2}$. (D) Inhibition of Bax translocation by the caspase inhibitor of Z-VAD fmk.
A

B

a
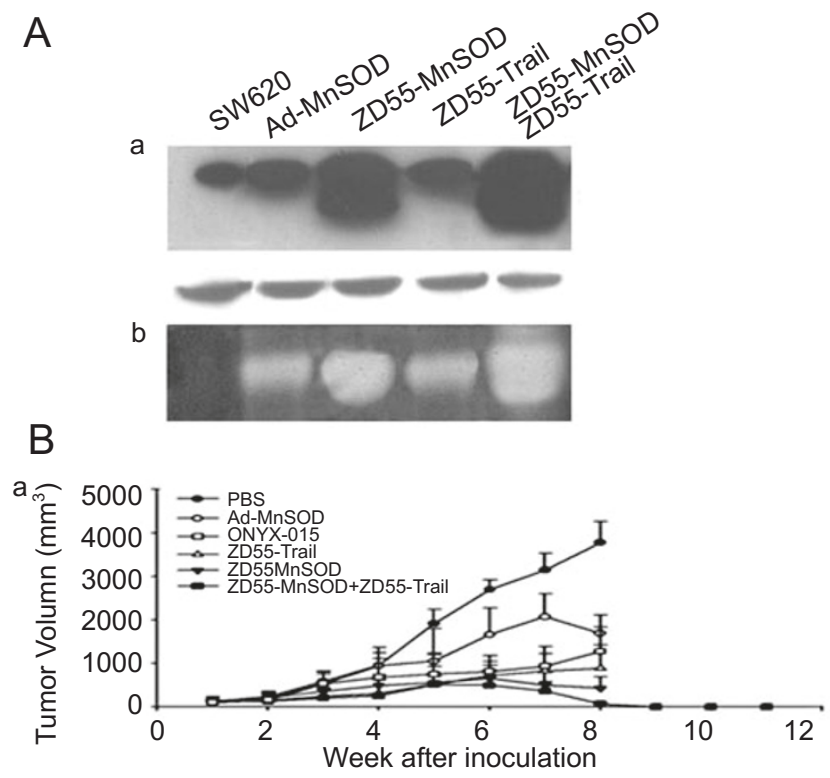

b

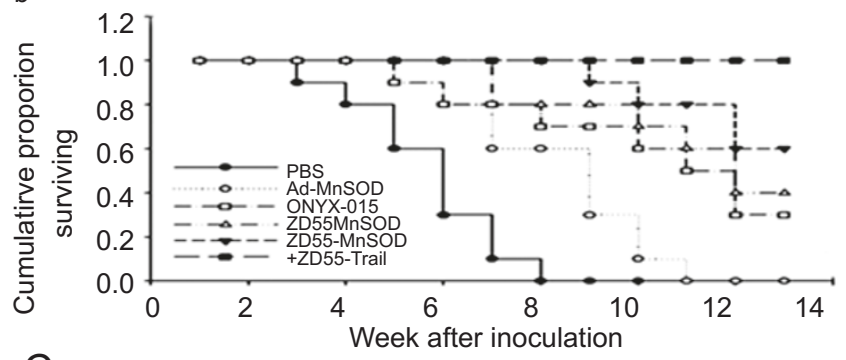

C

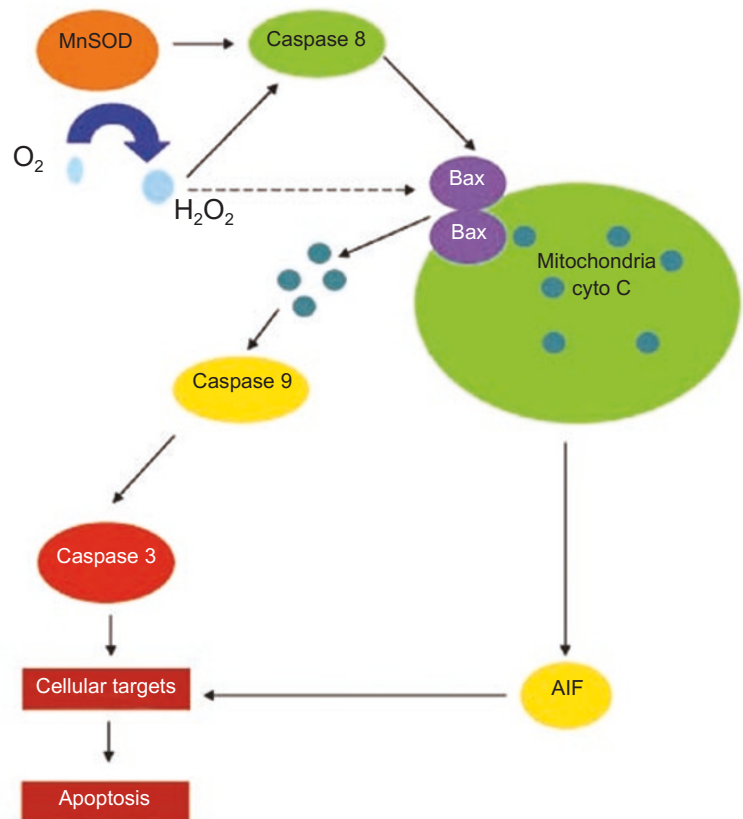

ZD55-MnSOD

Z-VAD-fmk

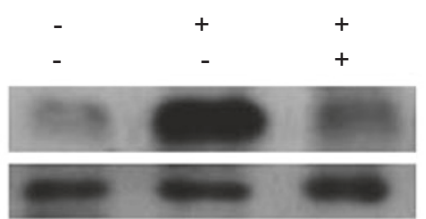

Bax actin 
exceed $1-2 \mathrm{~mm} 3$, they require penetration by blood vessels to supply blood and nutrition. TRAIL (or Trail) is the "TNF Related Apoptosis Inducing Ligand", one member of TNF superfamily, TNFSF10, specifically kills the transformed or cancerous cells but scarcely affects normal cells $[4,5]$. The Trail gene and K5 gene together have compensatory effects: TRAIL kills tumor cells by apoptosis, while K 5 blocks vessel branching and growth and in turn nutrition supplement. The combination of both genes as virotherapeutics should yield an effective antitumor effect. ZD55-Trail and Ad-K5 were constructed as in Figure 1A. Although Ad cannot replicate because it lacks the E1 region of adenovirus, it can replicate when the E1A and part of the E1B loci of ZD55 are intoduced. When the colorectal carcinoma SW620 was inoculated in nude mice at the right dorsal flank and treated with ZD55-Trail and Ad-K5 together, the tumor mass was completely eliminated [6] as shown in Figure 2.

A second pair used for virotherapy was ZD55-Trail and ZD55-Smac because these two genes have synergistic effects. The ZD55-Trail and ZD55-Smac were constructed as described in Figure 1A. After treatment of the hepatocellular carcinoma (HCC) BEL-7404 induced hepatoma by the combined use of ZD55-Trail and ZD55-Smac, the tumor mass was completely eliminated as shown in Figure $3 \mathrm{~A}[7]$.

The apoptotic activities of TRAIL are mediated by the activation of caspases 8 and 3. Hepatomas are more resistant to the apoptotic effects of TRAIL due to the expression of IAP (Inhibitor of Apoptosis) that blocks the activation of caspase 3 (Figure 3B). After inhibition of IAP activaty by Smac, the hepatoma becomes very sensitive to TRAIL's apoptotic effect (Figure 3B). In addition we can see that Trail will induce the release of Smac from mitochondria through the pathway of caspase8, Bid, Bak, and Bax. Smac increases the action of Trail and Trail induces the release of Smac. Therefore both genes have synergistic effect and the hepatoma was completely eliminated in all mice by the combined use of ZD55-Trail and ZD55-Smac. This strategy can be applied to the treatment of cancers in which IAP is overexpressed.

We found that ZD55-TRAIL induced superoxide dismutase (MnSOD) expression in SW620 cells as shown in Figure 4A. Accordingly ZD55-Trail and ZD55-MnSOD were combined to treat SW620 induced colorectal carcinoma; the tumor masses were completely eliminated in all mice as shown in Figure 4B.

MnSOD dismutates the free radical $\mathrm{O}_{2}$ - to form $\mathrm{H}_{2} \mathrm{O}$ and $\mathrm{H}_{2} \mathrm{O}_{2}$, which induces the translocation of Bax to the membrane and induces the expression of caspase 9 etc. We found that $\mathrm{H}_{2} \mathrm{O}_{2}$ induced the expression of caspase 8 as shown in Figure $4 \mathrm{C}$; the inhibition of caspase 8 by Z-VAD-fmk decreased the expression of caspase 8 (Figure 4D).

Using the Targeting Dual Gene-Virotherapy Strategy, we completely eliminated xenograft tumor mass in all nude mice in selected tumor models. We are confident that this technology may treat a wide variety of tumors in mice. Scientists previously used two genes to treat animal tumors, but they failed to completely eradicate all the tumors mass.

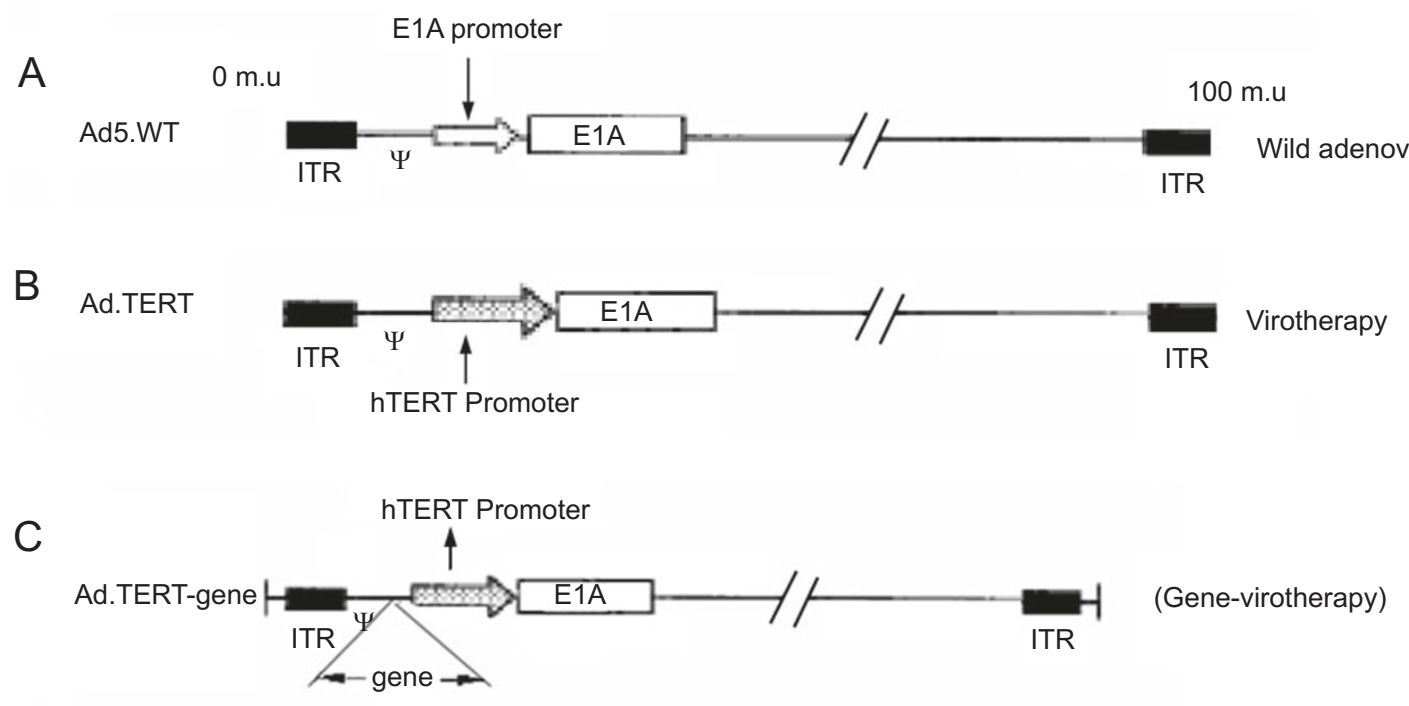

Figure 5 Construction of hTERT-Gene for antitumor therapy. (A) Wild type Adenovirus, its E1A was controlled by itself promoter. (B) hTERT promoter was used to replace E1A itself promoter to form Ad-hTERT. (C) An antitumor gene Trail expression cassette was inserted into Ad-hTERT. 
A

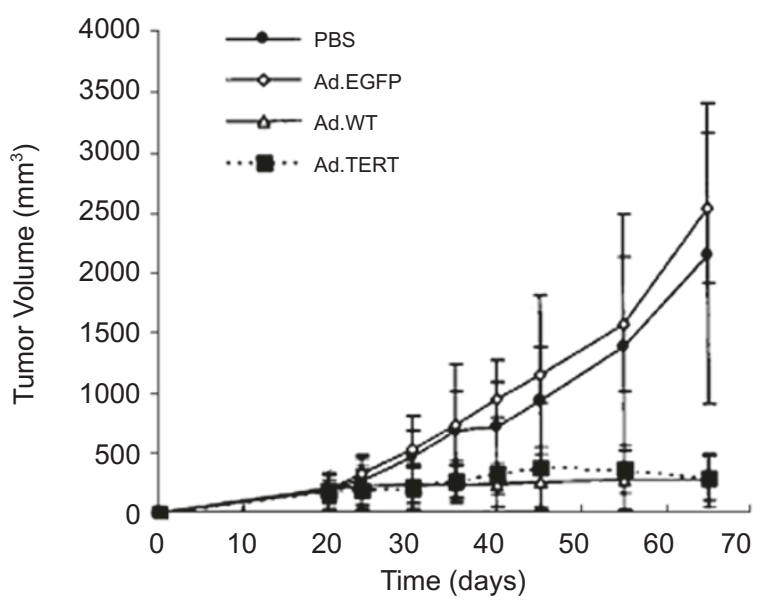

Hepatoma BEL 7404

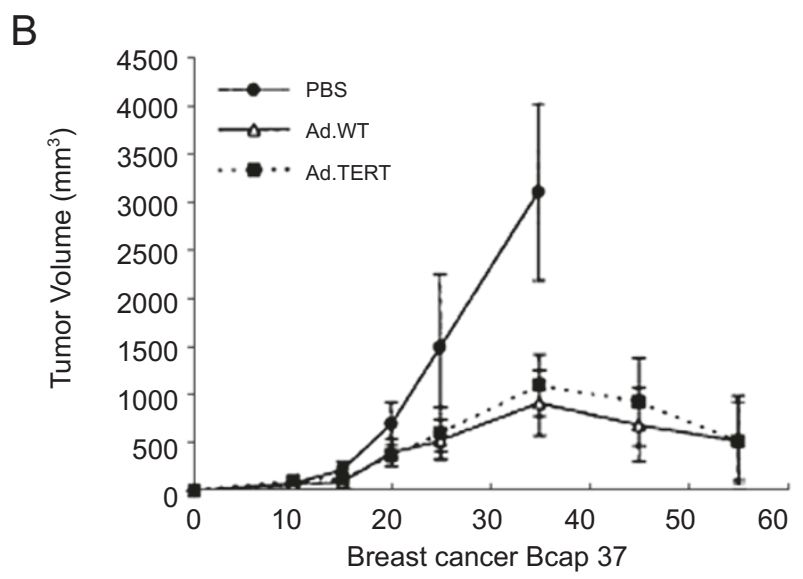

Figure 6 Antitumor effect of Ad-hTERT. (A) Antitumor effect of Ad-hTERT for Hepatoma BEL 7404. (B) Antitumor effect of AdhTERT for Breast cancer Bcap 37.

One reason may be that cancer specific replication vector was not used, while replication deficient adenovirus of routine gene therapy was used instead. The second reason may be that gene pairs with synergistic or compensatory activities were not used. With our strategy, we have data in numerous tumor transplant systems where tumor masses were eliminated. We now desire to develop an effective treatment regimen to treat patients. ZD55-IL-24 alone or in combination with ZD55-Trail is an excellent choice for clinical trials.

\section{hTERT system and the multiple cancer specific target- ing strategy}

hTERT is the human telemerase reverse transcriptase which is overexpressed in $85 \%-90 \%$ cancer. If the hTERT
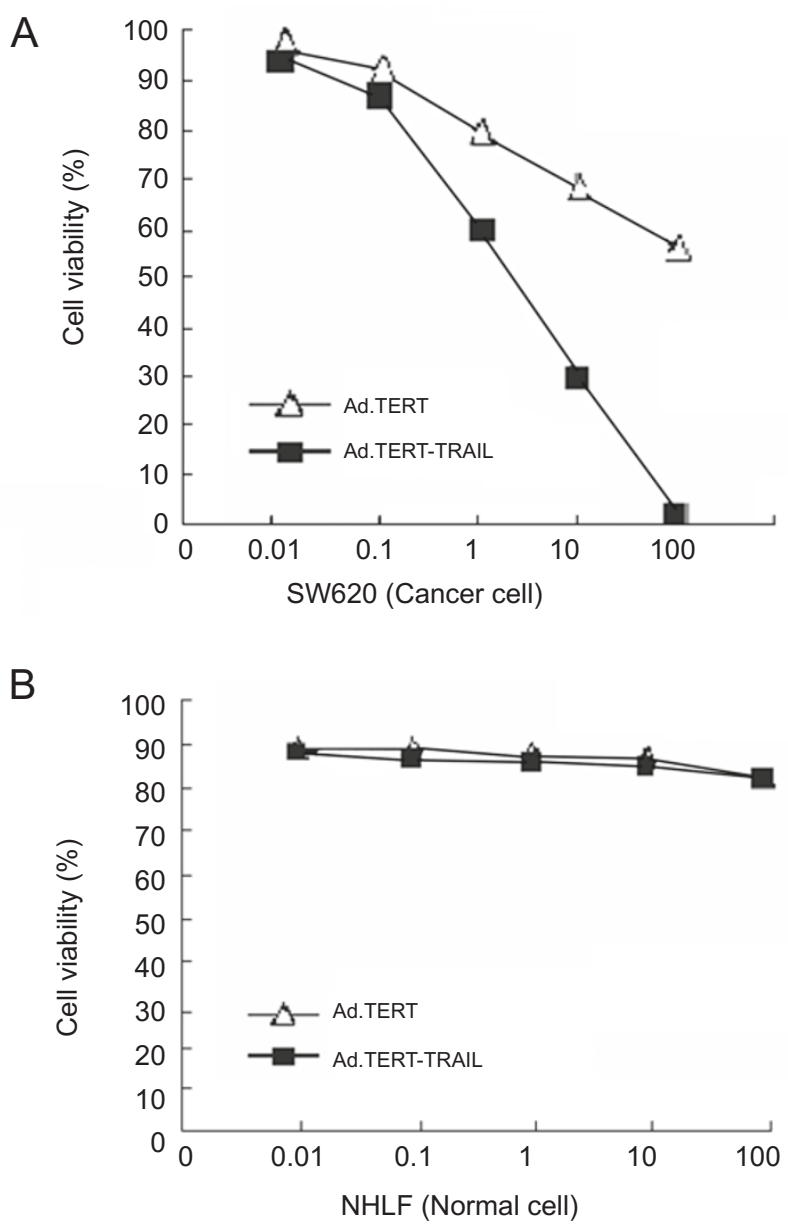

Figure 7 Antitumor effect of Ad-hTERT and Ad-hTERT-Gene. (A) Antitumor effect of Ad-hTERT-Trail and Ad-hTERT in colorectal carcinoma SW620. (B) Antitumor effect of Ad-hTERT-Trail and Ad-hTERT in Normal NHLF cell.

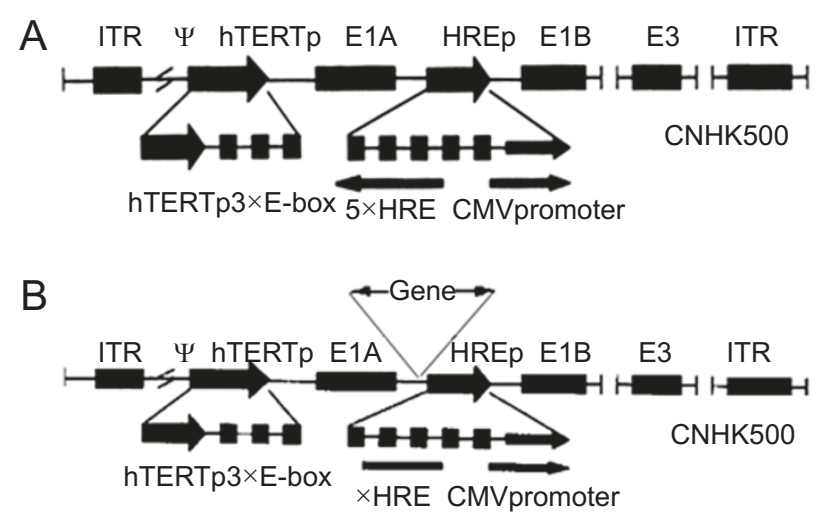

Figure 8 Construction of Multiple Cancer Specific Targeting Virotherapyor its Gene-Virotherapy. (A) Virotherapy with Ad-hTERTHRE. (B) Gene-virotherapy with Ad-hTERT-HRE-GM-CSF (or P53). 
promoter could be used to drive antitumor genes, the transgenes should be highly expressed specifically in malignent tumors.

In wild type adenovirus, its key gene E1A is controlled by its own E1A promoter (Figure 5A). After replacing the E1A promoter with the hTERT promoter, we created the cancer-specific targeting virus Ad-hTERT for targeting virotherapy (Figure 5B). An antitumor gene can be inserted into Ad-hTERT (Figure 5B) to create Ad-hTERT-Gene for targeting gene-virotherapy (Figure 5C).

The Ad-hTERT (or TERT-Adv) has broad targeting antitumor effect, in either hepatoma BEL-7404 or in breast cancer Bcap37 as shown in Figure 6A, B [8]. This is the second paper in literature using the human hTERT promoter to control E1A expression for the study of animal cancer treatment, and is the first paper to show the broad antitumor effect as shown in Figure 6 (A, B).

But it is only a virotherapy. If furthermore inserting an antitumor gene into Ad-hTERT to become Ad-hTERTGene (Figure 5C), the antitumor effect of Targeting GeneVirotherapy as Ad-hTERT-Trail is much better than AdvhTERT in SW620 cell, but is not toxic in normal NHLF cells (Figure 7A, 7B) $[9,10]$

\section{Multiple cancer specific targeting gene-virotherapy strategy}

The above work concerning hTERT underscored its potential in combination with another antitumor gene for multiple cancer specific targeting gene-virotherapy that will have more and substantial antitumor effect and utility.

HRE is the hypoxia factor HIF-1 binding element. In cancer, the expression of HIF-1 is highly increased. This element could be inserted into a promoter of a viral vector to achieve a cancer-specific upregulation of a given gene. After placing the HRE in the HTERT promoter to control E1A or E1B expression respectively, the resulting vector is an enhanced cancer specific targeting virus for therapy (Figure 8A). If we further insert an antitumor gene into this virus, it will become gene-virotherapy with enhanced cancer replicative targeting (Figure 8B). The GM-CSF cDNA has been inserted according to Fig. $8 \mathrm{~B}$ and was approved as a special case to enhance the immunological effect of cancer patients. In clinical trial, it has no obvious side effect but was an effective treatment for hepatoma patients ( $\mathrm{Au}$ : need references here). In Figure 8B, the p53 was also used in the place GM-CSF to become Ad-TERTHRE-p53 which has a more enhanced antitumor effect than Ad-TERT-HRE-GM-CSF and is applying for clinical use (data not shown). We created different cancer specific targeting gene-virotherapy drug for different special cancer treatment. For example, in hepatoma, we shall construct the Ad-TERT-AFP- Gene1-Gene2. Gene1 is a patented hepatoma specific suppressor gene under the control of hepatoma specific AFP promoter while Gene2 is tumor killer gene. The hTERT and AFP work together to drive the genes only in hepatomas and the Gene1 and Gene2 work together to completely eliminate the hepatoma mass in all mice. For colorectal cancer, we use a suppressor gene SP13 (with patent) and the CEA promoter.

\section{References}

1 Kirn D, Martuza RL, Zwiebel J. Replication-selective virotherapy for cancer: Biological principles, risk management and future directions. Nature Med. 2001; 7: 781.

2 Xin Yuan Liu. A new anticancer strategy - genetic and virological treatnent of cancer. Chinese J Cancer Biother 2001; 8: 1.

3 Zhao L, Gu J, Dong A, et al. Potent antitumor activity of oncolytic adenovirus expressing mda-7/IL-24 for colorectal cancer. Hum Gene Ther 2005; 16: 845-58.

4 Griffith TS, Chin WA, Jackson GC, et al. Intracellular regulation of TRAIL-induced apoptosis inhuman melanoma cells. J. Immunol. 1998; 161: 2833-40.

5 Pitti RM, Marsters SA, Ruppert S, et al. Induction of apoptosis by Apo-2 ligand, a new member of the tumor necrosis factor cytokine family. J Biol Chem 1996; 271: 12687-90.

6 Liu XY, Qiu SB, Zou WG, et al. Effective gene-virotherapy for complete rradication of tumor mediated by the combination of hTRAIL (TNFSF10) and plasminogen k5. Mol Ther 2005; 11: 531-41.

7 Pei Z, Chu L, Zou W, et al. An oncolytic adenoviral vector of Smac increases antitumor activity of TRAIL against HCC in human cells and in mice. Hepatology 2004; 39: 1371-81.

8 Zou W, Luo C, Zhang Z, et al. A novel oncolytic adenovirus targeting to telomerase activity in tumor cells with potent. Oncogene 2004; 23: 457-64.

9 Yun-xia Tang, Yu Chen, Jin-fa Gu, et al. Cancer gene-virotherapy of Ad-TERT-TRAIL. Chinese J Cancer 2005; 24: 536-42.

10 Zhang Q, Nie M, Sham J, et al. Effective gene-viral therapy for telomerase-positive cancers by selective replicative-competent adenovirus combining with endostatin gene. Cancer Res 2004; 64: 5390-97. 


\title{
Targeting gene-virotherapy of cancer
}

\author{
Xin Yuan $\mathrm{Liu}^{1,2}$, Jin $\mathrm{Fa} \mathrm{Gu}{ }^{1}$ \\ ${ }^{I}$ Institute of Biochemistry and Cell Biology, Shanghai Institutes for Biological Sciences, Chinese Academy of Sciences, 320 Yue \\ Yang Road, Shanghai 200031, China; ${ }^{2}$ Xinyuan Institute of Medicine and Biotechnology, School of Life Science, Zhejiang Sci-Tech \\ University, Hangzhou, 310018, China
}

Cell Research (2006) 16:25-30. doi:10.1038/sj.cr.7310005; published online 16 January 2006

Due to concerns regarding the overlapping figures in this review that are identical to those contained in a review article that we have co-authored and published earlier, we retract the above paper we published in Cell Research. We apologize for any confusion that may be caused by this matter, although we stand by the scientific contents contained in the Cell Research paper. 